\title{
SELF-REGULATED STAR-FORMATION IN GALAXIES
}

\author{
J.KÖPPEN, CH.THEIS AND G.HENSLER \\ Institut für Astronomie und Astrophysik, \\ Universität Kiel, Germany
}

In the chemodynamical models of galaxies the energy input from massive stars into the ambient medium results in a self-regulation of the star formation rate (SFR). A thorough analytical and numerical study of this model shows that there is always a strong and negative feed-back, and the SFR becomes almost independent of the assumed stellar birth function (SBF). The time-scale to reach this equilibrium is much shorter than the gas consumption time-scale, hence the models evolve along this solution for most of the time. This mechanism provides a physical explanation for a quadratic dependence of the SFR on gas density. For more details cf. Köppen et al. (1995), A\&A 296, 99 and in preparation.

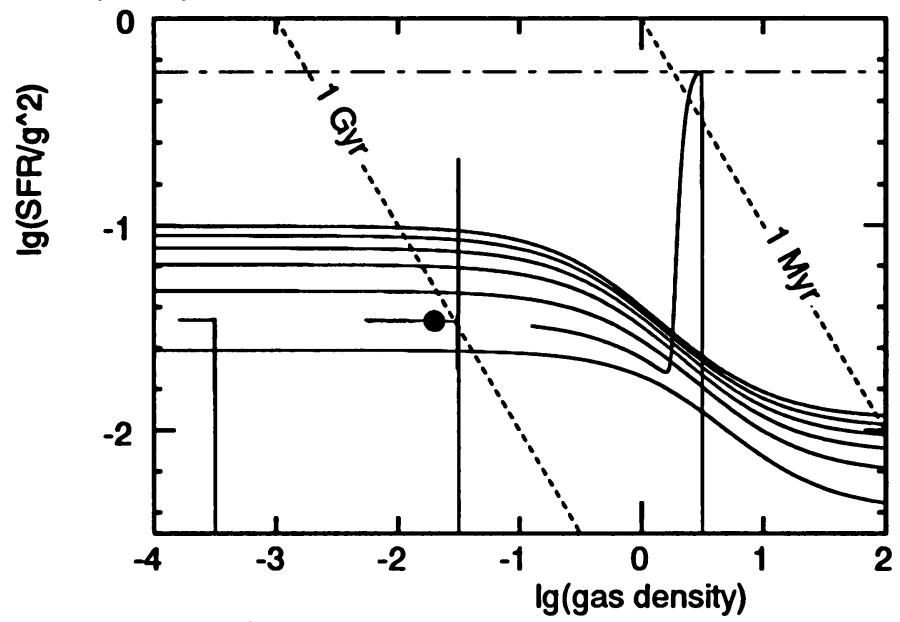

Figure 1. The ratio SFR $/ g^{2}$ of the star-formation rate and gas density as a function of the gas density $g$ (in $\mathrm{M}_{\odot} / \mathrm{pc}^{3}$ ): shown are the analytical equilibrium solutions for various values of the constant of proportionality in the SBF $(\log C=9,7, \ldots,-1$ from top to bottom). Also depicted are results from three numerical models with initial gas densities of $\log g=0.5,-1.5$, and -3.5 . Lines of equal star-formation timescale are short-dashed. The filled dot marks the equilibrium for our 'standard' value $C=0.55$ at the density of the local interstellar medium $\left(n_{\mathrm{gas}}=1 \mathrm{~cm}^{-3}\right)$. 